\title{
UNIVERSIDAD DE COSTA RICA: PREMISAS, POLÉMICAS Y PROPUESTAS ACTUALES EN EDUCACIÓN
}

\author{
María Pérez Yglesias ${ }^{1}$
}

\author{
Recibido 15-VII-2002 • Aceptado 13-VIII-2002
}

\begin{abstract}
Resumen: El artículo busca sintetizar algunas reflexiones sobre las viejas y nuevas premisas y polémicas educativas que se plantean y discuten en este principio de milenio, con el propósito de pensar cómo la Universidad de Costa Rica responde a las exigencias y expectativas internas y externas de los últimos años, haciendo énfasis en la investigación y el posgrado en relación con la docencia y la acción social.

Sin pretender ser exhaustivos se trata de responder, con ejemplos, si el modelo de la Universidad de Costa Rica se transforma con acciones concretas y con una perspectiva educativa coherente y académica. Como se dice en el planteamiento "hemos decidido reunir algunos tópicos en torno a cuatro grandes temas que incluyen distintos aspectos de los planteados anteriormente: por una parte el modelo de organización académica; en segunda instancia la apertura a un trabajo dialógico, en equipo, interdisciplinario e interinstitucional; en tercer lugar la incursión en nuevas tecnologías y en educación permanente y por último la tendencia hacia una Universidad cualitativa con impacto social".
\end{abstract}

Palabras clave: Educación, Universidad, Investigación, Educación Permanente, Estudios de Posgrado.
La participación como expositora en el Seminario-Taller Regional: Experiencias, tendencias y alternativas en el desarrollo de los estudios de posgrado y la investigación ${ }^{2}$ me lleva a reflexionar sobre varios tópicos relativos a la propuesta de una Educación Superior para el siglo XXI. Esa propuesta, que se prepara como un reto mundial con talleres regionales y, ya en 1998, con la Conferencia Mundial de la Educación de la UNESCO en París y la Conferencia en Belgrado en 1999, se concreta en algunos lineamientos al futuro.

Las discusiones y los textos sobre educación se multiplican en los albores de este tercer milenio y la educación sigue en la lid a pesar de las globalizaciones; los posmodernismos; las políticas liberales y privatizantes; la explosión de las torres gemelas de New York; la guerra contra el terrorismo y a favor de un liderazgo único de los norteamericanos; los bombardeos en Afganistán, Bin-Laden y la CIA; la situación palestino-israelí; la implementación del euro como moneda europea; los países divididos en naciones; las confrontaciones religiosas e ideológicas; los desastres naturales y los cambios climáticos; las luchas entre hermanos y vecinos por territorios y poderes; el miedo a perder la identidad, al 
cambio radical o al fin del mundo; el aumento de la pobreza, la injusticia, la iniquidad y la violencia; la sofisticación informática, armamentista y espacial o el mundial de fútbol en Corea...

Los seres humanos aún tenemos la esperanza de conquistar el planeta con ideas y no con armas, con descubrimientos científicos que mejoren la calidad de vida y no la destruyan, con generación de conocimientos y no con imposiciones económicas, con unidad que respete las diversidades culturales y no con homogeneización, con sueños y creatividad y no con reiteraciones y rutinas sin fin.

Por eso seguimos trabajando en nuestros insignificantes espacios -si se piensan en milenios y en geografías- creyendo en que la formación de cada persona, la forja de su identidad, la ponderación de sus habilidades, aptitudes y actitudes positivas en torno a los saberes para vivir mejor, podrán cambiar la tierra palmo a palmo, lograrán convencer idea a idea para transformar esta realidad agónica, en un mundo mejor para las colectividades.

Por eso nos aferramos a las posibilidades y las potencialidades de nuestras instituciones universitarias, de nuestros académicos, de nuestros estudiantes y profesionales.

Por eso privilegiamos el trabajo en equipo, la capacidad crítica, la excelencia y la ética, la investigación como base de una docencia de calidad y una acción social efectiva con gran creatividad.

Por eso creemos en el poder del conocimiento $^{3}$, de la generación del conocimiento y la aplicación del conocimiento, en el poder de la comunicación, de los valores humanos y de la educación.

El trabajo que aquí se propone no busca la exhaustividad, ni siquiera pretende un acercamiento equilibrado y coherente a todos los ámbitos en que se desenvuelve y transforma, cada día, la Universidad de Costa Rica. Se trata de sintetizar unas reflexiones generales sobre las propuestas educativas (algunas utópicas, otras más concretas) que se debaten o intuyen para el siglo XXI y de pensar cómo nuestra institución responde a las exigencias y expectativas internas y externas en los últimos años, con énfasis en la investigación y el posgrado.

\section{De las oposiciones a los complementos}

La apuesta por el poder del conocimiento frente a otros poderes, para regir el futuro, centra el interés en la educación, el desarrollo de la ciencia y la tecnología, la preparación humanística y las metodologías de enseñanza, además de la propuesta de planes curriculares flexibles y contextuales.

De una clara propuesta donde se privilegian la calidad, la pertinencia, la organización y administración eficiente y la colaboración internacional, de un espacio donde la ética y el respeto a las diferencias potencian las transformaciones, se pasa a discutir temáticas concretas y problemáticas fundamentales como lo son la protección del medio ambiente y el desarrollo sostenible y sustentable, la equidad de géneros, la cultura de la paz, el respeto a la diferencia (unidad en la diversidad)...

Estos planteamientos, como líneas invisibles, impulsan los sueños de una educación de excelencia para todos y todas, de una educación que abra puertas al empleo, que rompa los horizontes de la creatividad, que mejore las relaciones entre los pueblos de culturas distintas y entre las personas, que genere espacios de ocio y entretenimiento complementarios, que permita una mejor calidad de vida material y espiritual, que convenza de la necesidad de seguir aprendiendo y actualizándose para toda la vida.

Las expectativas que fundan y fundamentan las discusiones sobre educación a lo largo y ancho del mundo, se quiebran con preocupaciones manifiestas 
en distintos foros que enfatizan en otras problemáticas paralelas. Estas preocupaciones, de gran actualidad, se han mantenido en el tapete desde hace muchos años.

A través del tiempo las visiones, misiones o propuestas educativas se han visto muchas veces como dicotomías, como oposición de contrarios que no lo son. El maniqueísmo, los absolutos, la objetividad, el causalismo, el blanco y el negro de los contrarios cede cada vez más, a la lógica de los grises, de las combinaciones, de los predominios, de la multicausalidad, de los procesos antes que los resultados, de la productividad frente a los productos listos para el intercambio y el consumo.

La inteligencia se valora introduciendo lo emotivo, la afectividad; la oposición de generaciones, de géneros, de etnias, de clases sociales se desgasta; la honestidad se privilegia sobre la objetividad y se valoriza lo subjetivo; lo cualitativo significa y lo cuantitativo se apoya con los análisis e interpretaciones. La relatividad de lo posmoderno se ajusta y el juego, la posibilidad, la utopía caen en el campo de lo posible. El científico asume el alma intuitiva, curiosa, aventurera del poeta y el poeta interpreta mundos con la lucidez, el trabajo y la tenacidad de un investigador humanista y profundo.

Cada una de nuestras premisas, que resume una preocupación en torno al proceso educativo, se presenta como una oposición que solo significa un continuum, un predominio sujeto a un momento histórico, un espacio geográfico, un énfasis o una propuesta particular o institucional. Ninguna de estas premisas constituye un planteamiento autónomo, ni completo, puesto que todas, o casi todas, se relacionan entre ellas: se apoyan, se oponen, se neutralizan pero, generalmente, se complementan. Plantearlas, una a una, solo busca introducir ideas clave que, en la tercera parte del trabajo, apoyan o explican algunos de los cambios y transformaciones que percibimos en la Universidad de Costa Rica.
Los ejes de discusión pueden percibirse desde dos niveles: en el primero se toman en cuenta las relaciones de la educación nacional con la internacional; en el segundo las relaciones específicas en nuestra institución. El contexto necesariamente incide y atraviesa los planteamientos y las polémicas dentro de la Universidad de Costa Rica ${ }^{4}$.

\section{De las premisas en discusión}

- Educación pública - educación privada. Esta dicotomía se presenta como una clara consecuencia de las discusiones y los cambios en torno a la función del estado nacional y la tendencia a la liberalización y la privatización en todos los niveles de acuerdo con patrones internacionales. En la Universidad de Costa Rica se discute sobre la actitud que debe asumir la institución frente a la eclosión de universidades privadas e, internamente, se polemiza sobre si la venta de servicios implica privatizar parcialmente o crear una "universidad paralela" o si se trata de una actividad complementaria a las funciones sustantivas de una universidad pública.

- Educación superior - otros niveles de la educación, con dos claras tendencias: la que propone fortalecer en los países en desarrollo la enseñanza primaria, la secundaria y algunos niveles básicos de la educación superior, dejando la investigación de punta y el posgrado para el primer mundo y la que propone un avance más equitativo entre los países, fortaleciendo los estudios superiores de alto nivel y la investigación en el tercer mundo. La primera propuesta es bastante recurrente en la Conferencia Mundial sobre Educación de la 
UNESCO (París, 1998). Ya desde mediados de la década de los setenta, la UCR crea el Instituto de Mejoramiento para la Educación Costarricense (IIMEC) y participa activamente como formadora de formadores y en las instancias que así se lo demandan en pro del mejoramiento educativo. La UCR fortalece, sobre todo a partir de la creación de Centros e Institutos de Investigación y del Sistema de Estudios de Posgrado en los años setenta, las especialidades, maestrías y doctorados y la investigación de alto nivel en todos los campos del conocimiento.

- Educación informal - educación formal. Se pasa de la discusión de los medios de comunicación de masas como mecanismos de educación informal al uso de las nuevas tecnologías de la información y la comunicación (NTIC) por medios informáticos. En este sentido la UCR ha participado activamente en la propuesta de Internet (Internet II) y ha mejorado sustancialmente sus equipos de informática.

- Educación virtual - educación presencial (escolarizada). Se ataca o defiende la educación a distancia y la enseñanza virtual como un medio para ampliar las posibilidades de enseñanza. La polémica se abre en torno a la iniquidad que produce la falta de acceso a los medios informáticos (tienen un alto precio, el mantenimiento es oneroso y caen en desuso rápidamente), por lo que parece contradictoria la premisa de "una educación virtual para todos". Además preocupa la falta de hábitos para la autoeducación y, sobre todo, la pérdida del contacto humano, la discusión cara a cara, la sensibilización y capacidad crítica.
La UCR tiene algunos Posgrados semi-presenciales e incursiona en la educación a distancia como un medio de ponderar la educación continua o para toda la vida.

- Educación terminal - educación para toda la vida. Actualmente se discute, incluso, si los títulos no deben tener un tiempo de pertinencia, se habla de recertificaciones profesionales que exijan la actualización permanente y se propone que las universidades asuman el reto de ser universidades abiertas, donde las personas puedan seguirse preparando el resto de sus vidas. El Sistema de Estudios de Posgrado ofrece la posibilidad de continuar empadronándose una vez que finaliza su programa, se formulan gran cantidad de cursos especiales de posgrado con diversas instituciones y se amplía la oferta de cursos de capacitación y actualización de conocimientos o teorías y metodologías.

- Educación cuantitativa - educación cualitativa. La calidad se marca como un hito fundamental y se enfrenta al acceso y la participación de todos y todas a los niveles universitarios sin discriminación de ningún tipo. En algunos países se opta por mantener universidades masivas que difícilmente pueden responder a un alto nivel académico y afianzar la investigación y la acción social. La UCR, a pesar de que intenta ampliar sus cupos de admisión para responder mejor a la amplia demanda, lo hace de manera racional sin perder la pertinencia, la calidad y la excelencia. Su vocación hacia la investigación y la acción social y no solo hacia la docencia, la coloca en una tendencia hacia la universidad cualitativa. 
- Educación informativa (trasmitir conocimientos) - educación formativa (forjar actitudes). Desde principios del siglo pasado esta es una discusión que permite plantear una serie de propuestas sobre educación: nueva educación, educación participativa, educación activa, educación centrada en el alumno, educación para la vida... La propuesta de quitar lo superfluo de los planes curriculares, favorecer lo contextual y asumir un punto de vista transdisciplinario lleva a la UCR a privilegiar la formación.

- Educación libre de evaluaciones - educación acreditada. Existe una política clara hacia la autoevaluación, la evaluación y la acreditación externa (nacional e internacional) en la que, cada vez más, se interesan las instituciones de educación superior. Esta necesidad de diferenciar los espacios por calidad, responde también a una necesidad de rendir cuentas a la sociedad sobre el quehacer universitario. En este momento la UCR se evalúa externamente ya sea en el país con SINAES (para acreditar carreras) y el ENA (para acreditación de laboratorios), como a nivel centroamericano (SICAR y SICEVAES del CSUCA) y organismos canadienses de acreditación. Además la institución posee un Centro de Evaluación Académica para el plano docente y se generan recientes procesos de evaluación de los Centros e Institutos de Investigación, los Posgrados y las publicaciones periódicas.

- Educación nacional - educación internacional. Cada vez más se plantean posibilidades de intercambio, colaboraciones de doble vía a nivel de investigación y docencia, títulos compartidos, trabajo en redes, consorcios, confederaciones de universidades... La UCR a través de la Oficina de Asuntos Internacionales y Cooperación Externa y algunas instancias dentro de la Investigación y el posgrado, se interesa por el funcionamiento de los convenios, el trabajo en redes como las ALFA, o el proyecto ANUIES-CSUCA, Consorcios como el CILPIC formado por catorce universidades y Centros de Investigación Latinoamericanos. Formamos parte de la AUIP, CINDA, OUI (Colam, IGU, IGLU) y, en este momento, se trabaja con programas de posgrado compartidos entre dos o más universidades estatales.

- Educación especializada - educación general o educación científico tecnológica - educación humanista. Esta vieja polémica sigue estando en boga, sin embargo, en los últimos tiempos se privilegia la formación general humanista y contextual como base y fundamento de la especialización y de la profundización en áreas científico técnicas. La discusión enfrenta los Estudios Generales como una propuesta a la base (horizontal) o vertical (durante los estudios), pero no niega la importancia de ser humanistas, conocedores del contexto nacional y regional e impulsores de una cultura general, además de la especialización.

- Educación disciplinaria - educación interdisciplinaria (multidisciplinaria o transdisciplinaria). En un mundo invadido por una información al instante, una creatividad multiplicada en todos los ámbitos, descubrimientos y transformaciones rápidas y posibilidades casi infinitas de acumular conocimientos, la disciplinaridad, limita, fragmenta, parcela 
o dificulta la comprensión de los procesos y la adaptación a las nuevas exigencias del estudio, el empleo e incluso las relaciones humanas. En la UCR teóricamente se privilegia el trabajo en equipos de investigación interdisciplinarios y se busca un trabajo más bien transdisciplinario. Muestra de ello son los Programas Institucionales de Investigación y las maestrías y doctorados multi o transdisciplinarios.

- Educación basada en la docencia - educación que privilegia, además, la investigación y la acción social. Tradicionalmente existe una preocupación en torno a la preeminencia de la parte docente sobre la investigación y la acción social. Nadie niega la importancia de las universidades como formadoras de profesionales de alto nivel; sin embargo, no tomar en cuenta las otras dos variables significa negar la vocación generadora de conocimientos y promotora de cambio social propia de una Universidad y situarse en el nivel de institutos técnicos de enseñanza superior o, lo que sería peor, en "transmisores de información".

- Educación según posibilidades intelectuales - educación por cuotas de género o marcada por edades, procedencias de grupo social, región, nacionalidad... La admisión a la UCR se realiza mediante criterios académicos, basada en la trayectoria del estudiante y el examen propiamente dicho. La selección no toma en cuenta ningún criterio discriminatorio, ni siquiera en cuanto a la edad como en otras universidades. Se discute la posibilidad de incluir criterios de procedencia (rural) y se trata de estimular en ciertos espacios una mayor participación por género ${ }^{5}$.
- Educación centralizada - descentralizada o desconcentrada, a nivel nacional o dentro de las instituciones mismas. La UCR se ha preocupado por acreditar como regionales sus Posgrados en la región centroamericana, bajo criterios de calidad y pertinencia. Actualmente se discute una mayor autonomía y eliminar el nombre de regional a las diferentes sedes de la misma Universidad. En los últimos años se favorece la autonomía relativa entre sedes y se busca ampliar el radio de acción con los recintos, a la vez que se busca una mejor distribución de recursos humanos, curriculares y de infraestructura.

- $\quad$ Educación de grado - educación de posgrado (preeminencia o equilibrio). La UCR fortalece sus estudios de grado antes de crear en 1976 el Sistema de Estudios de Posgrado. Desde el inicio trata de mantener un sano equilibrio.

\section{La UCR: ¿modelo en transformación?}

En el documento del Consejo Universitario, Principios y políticas generales de la Universidad de Costa Rica, 1997 "se replantean las políticas universitarias para reflejar fielmente en ellas el espíritu del Estatuto Orgánico" y se señala la necesidad de que la institución garantice una educación integral, que cubra lo ético-cultural, científico tecnológico y económico social, basada en los principios de excelencia, solidaridad y compromiso, transparencia, humanismo, participación, austeridad y desconcentración (CU 1997, 3-5-7).

Ante la imposibilidad de desarrollar cada una de las premisas anteriores y su respectiva discusión dentro de la Universidad de Costa Rica a la luz de sus principios 
generales, hemos decidido reunir algunos tópicos en torno a cuatro grandes temas que incluyen distintos aspectos de los planteados anteriormente: por una parte el modelo de organización académica; en segunda instancia la apertura a un trabajo dialógico, en equipo, interdisciplinario e interinstitucional; en tercer lugar la incursión en nuevas tecnologías y en educación permanente y por último la tendencia hacia una Universidad cualitativa con impacto social.

\section{Hacia una universidad cualitativa}

La década de los setenta marca para la Universidad de Costa Rica y para la educación superior universitaria un camino a seguir. Varias universidades públicas europeas y latinoamericanas optan por abrir sus puertas a miles de estudiantes sin pruebas de admisión, ni controles académicos, administrativos y del entorno, que permitan mantener una alta calidad en los estudios. La masificación universitaria o la opción por una universidad cuantitativa marca algunas políticas gubernamentales sobre el financiamiento de la educación superior que aún hoy repercuten negativamente en ciertos aspectos, por ejemplo: repartir el presupuesto para las universidades públicas de acuerdo con el número de estudiantes sin darle valor real a la investigación, las necesidades de infraestructura o los programas de acción social o de vinculación con el sector productivo público o privado.

La Universidad de Costa Rica crece en estudiantes y en personal académico, para suplir las necesidades docentes y de los nuevos espacios de investigación que se multiplican, pero lo hace tomando en cuenta su capacidad instalada, sus posibilidades docentes reales y la necesidad de no dejar a un lado ninguna de las funciones sustantivas de la institución.

En los setenta, una década de explosión demográfica en los niveles de educación superior, la UCR opta, en el Tercer Congreso Universitario (1973), por ampliar su radio de cobertura a diferentes regiones del país y funda los Centros Regionales, al mismo tiempo que funda la Facultad de Ciencias Sociales, crea las Vicerrectorías y abre, en 1975, el Sistema de Estudios de Posgrado, adscrito a la Vicerrectoría de Investigación, donde también se inserta el Sistema de Bibliotecas y de Información (SIBDI) y la Dirección Editorial (DIEDIM) indispensables para consolidar el espacio investigativo.

Es en los años setenta que se crean la Universidad Nacional (UNA), el Instituto Tecnológico de Costa Rica (ITCR) y la Universidad Nacional de Educación a Distancia (UNED). Es también en esa década cuando se inicia la explosión de instituciones de educación superior de carácter privado, con la Universidad Autónoma de Centroamérica (UACA).

En la segunda mitad de los setenta cristaliza la propuesta de establecer un Consejo Nacional de Rectores (CONARE) que vele por las políticas institucionales y la acreditación de los planes de estudio de las universidades públicas. La UCR participa en la Confederación de Universidades Públicas de Centro América (CSUCA), desde su creación y con el tiempo se le suman las otras tres universidades estatales.

La UCR, pionera por su experiencia de más de seis décadas y su compromiso con la investigación y una acción social de impacto, al fortalecer la investigación interdisciplinaria en Centros de Investigación y al pensar en los estudios de posgrado como Sistema marca su preferencia hacia una universidad más cualitativa que cuantitativa

La investigación y la acción social se fortalecen y ya en la segunda mitad de los noventa, casi el $70 \%$ de la investigación nacional y el 50\% de la regional está en manos de la UCR, gracias al desarrollo de los espacios de investigación, la política de enviar estudiantes a sacar posgrado al exterior, 
las políticas y lineamientos, a la capacidad de establecer relaciones de colaboración (convenios, proyectos, redes), con investigadores e instancias de investigación internacionales.

El SEP nace en 1975 con un doctorado (Filosofía) y cuatro Maestrías Académicas y no es sino hasta mediados de los años ochenta que las Especialidades Médicas entran a formar parte del Sistema en convenio con el CENDEIS, brazo académico de la CCSS y se trabaja con otras especialidades en salud y en derecho. En la década de los noventa (1993) y por una coyuntura particular, la Universidad se abre a la propuesta de Maestrías Profesionales y nuevos Doctorados Académicos, que sin duda vienen a fortalecer el ámbito de la investigación y el intercambio académico ${ }^{6}$.

Las Maestrías profesionales surgen como una necesidad frente a la imposibilidad de reconocer y equiparar títulos del exterior con ese carácter. El SEP los asimila a Especialidades y, en ocasiones, el Consejo Universitario resuelve positivamente las apelaciones y las equipara con Maestrías Científicas, sin cumplir con los requisitos mínimos de investigación. Por otra parte existe una fuerte presión en ciertos campos profesionales que desean profundizar conocimientos y hacer trabajos aplicados y no una tesis propiamente dicha. Aunque en CONARE se discute fuertemente que se trata de "programas de posgrado basados en cursos", lo cierto es que prevalece la opinión de la UCR de que en todos los estudios de posgrado, no importa si se trata de especialidades, maestrías profesionales o académicas o de doctorados, la investigación es indispensable. ${ }^{7}$ Se amplían las opciones profesionales sin detrimento de la investigación. En el año 2001 se organizan jornadas de autoevaluación en las comisiones de posgrado y en el 2002 se realizan las institucionales. En este año los programas de posgrado regionales están en proceso de acreditación del SICAR (CSUCA) con evaluadores externos a la Universidad ${ }^{8}$.
El Centro de Evaluación Académica, adscrito a la Vicerrectoría de Docencia, ha dado pasos importantes en las estrategias de evaluación de los académicos a nivel docente y de los planes y programas de estudio. Además, la Universidad se ha involucrado en procesos de acreditación nacionales, más allá del de CONARE, a través del SINAR (Sistema Nacional de Acreditación) que acredita las Escuelas de Medicina y Trabajo Social; de organismos internacionales como el canadiense que acredita Ingeniería Civil, Industrial y Eléctrica; el SISEVAES que busca la acreditación para las carreras de universidades que forman parte del CSUCA.

La necesidad de fortalecer la crítica académica lleva a la Vicerrectoría de Investigación (VI) a desconcentrar, en los Consejos Científicos de Centros e Institutos de Investigación y en las comisiones de investigación de las Escuelas y Facultades, los procesos de presentación de proyectos, evaluación de informes parciales y finales y prevé la existencia de pares externos para la evaluación cuando se considera necesario. En el año 2000 una comisión de académicos inicia en la Vicerrectoría de Investigación, la propuesta de evaluación para mejoramiento.

La búsqueda de una estrategia de autoevaluación y evaluación, para todo el espacio investigativo, que permita tomar decisiones, mejorar las debilidades y aprovechar las fortalezas ha sido una política constante de la VI en los últimos cuatro años. La evaluación permite dar seguimiento, cerrar Centros, programas o proyectos no activos, exigir un determinado rendimiento o apoyar la transformación de un programa en Centro o Instituto o crear una nueva Unidad Especial de Investigación. La evaluación se aplica como plan piloto a los Centros, Institutos, Estaciones Experimentales y se trabaja, arduamente, en la evaluación de las revistas universitarias. El esfuerzo pretende, igual que en el CEA y el SEP, forjar el hábito de la rendición de cuentas y mejorar la calidad en todos los niveles . 
La falta de identificación institucional, la carencia de estímulos para quienes son universitarios por opción e interés, la sensación de que no se premia a quien lo merece y se pone techo a los académicos más productivos, los salarios poco competitivos y la falta de otros incentivos, las trabas y la ineficiencia de ciertas oficinas administrativas poco flexibles son algunas de las críticas que se escuchan, más a menudo, en los pasillos de la institución.

\section{Una propuesta triádica: Investigación, docencia y acción social}

La organización en áreas académi$\operatorname{cas}^{10}$, Facultades y Escuelas ${ }^{11}$ data desde los orígenes de la Universidad de Costa Rica, que se funda hace 62 años (1940). En los Consejos de Área y fundamentalmente en las Asambleas de Escuela y de Facultad, se concentra la dirección de las tres funciones académicas básicas, de la administración y la atención de los estudiantes hasta 1973, en que, con la creación de las cinco Vicerrectorías $^{12}$ en el Tercer Congreso Universitario, algunas de las funciones sustantivas se reorganizan y comparten.

A partir de esos años y con el cambio del Consejo Universitario de un Consejo de Decanos a un órgano de elección ${ }^{13}$, se desata en la Universidad una discusión sobre las potestades de los Directores y Decanos, la descentralización y centralización de los procesos, la pérdida de competencias de las comisiones de acción social y de investigación, los concursos de antecedentes, los planes de trabajo de los profesores, etc. A esta discusión se suma la de las relaciones entre los Centros Regionales, ahora Sedes y la Sede Rodrigo Facio y la necesidad o no de desconcentrar y/o descentralizar el poder en manos de los Directores y Asambleas de Sedes.

Si se hace un balance de la relación de las Vicerrectorías y las Escuelas y Facultades es fácil evidenciar, por una parte, que sigue existiendo un gran poder en manos de las Asambleas, de las direcciones y decanatos y de las comisiones. Es el poder del presupuesto, la contratación, el manejo administrativo y académico de los planes de estudio y de todas las decisiones de vida cotidiana.

A cada uno de los Vicerrectores le corresponde, además de asumir con el Rector su ámbito de competencia, la responsabilidad de mantener la información completa y organizada, darle seguimiento a los órganos que tiene adscritos, realizar un balance para el mejoramiento, rendir cuentas y mantener la calidad, organizar espacios de intercambio entre sus órganos y las otras instancias, actuar como mediador de conflictos y apoyar, promover, fortalecer, mejorar, asesorar, evaluar, estimular... las actividades pertinentes.

A pesar de la concepción tripartita de la Universidad de Costa Rica, el presupuesto, salvo excepciones en algunas áreas, se adjudica primero a los cursos e incluso a las comisiones de distinta índole y un mínimo se utiliza en investigación y acción social (en ese orden). Si se hacen recortes presupuestarios o surgen nuevas necesidades, en general perjudican los proyectos de investigación o de extensión. Los concursos se ganan como plazas docentes, raramente en espacios de investigación y nunca como acción social. Asimismo los tiempos son distribuidos por las Escuelas y Facultades y por la Vicerrectoría de Docencia; solo como excepción existen unos pocos para cubrir necesidades perentorias de Centros e Institutos. Varios investigadores y asistentes son financiados con los recursos de los programas, proyectos y actividades de investigación.

En el caso del Sistema de Estudios de Posgrado los tiempos son distribuidos por las Escuelas y Facultades; pero existe un número de plazas que se manejan directamente desde la Decanatura y que se utilizan para apoyar los Programas que lo necesiten. A partir del 2000 se ha dado un 
aumento progresivo de plazas como un acuerdo para fortalecer el SEP, por parte de la Rectoría y el Consejo Universitario ${ }^{14}$. Hasta 1993 solo existían programas de posgrado con financiamiento regular y algunos que trabajaban con convenios institucionales o aportes externos. Ese año se abre la modalidad de programas de posgrado "autofinanciados", más bien programas con financiamiento externo o complementario, por la clara inversión que la Universidad hace en aulas, personal administrativo, luz, agua, limpieza, uso de laboratorios y equipo... El costo del crédito lo fija el Consejo Universitario (actualmente 15.000 colones, más- menos $30 \%$ ) y se maneja por medio de fondos especiales ubicados en la Oficina de Administración Financiera (OAF). Cada programa paga a la Universidad entre un 5 y un $8 \%$ por administrar su fondo restringido y aporta entre un 10 y un $20 \%$, según sus condiciones particulares, al fondo común o fondo solidario, que se ocupa de redistribuir estos recursos para mejorar las condiciones del posgrado como un Sistema ${ }^{15}$. Esta modalidad permite el pago de los cursos y/o de complementos salariales por coordinaciones, docencia y dirección o asesoría de investigaciones y hace posible abrir el Sistema a propuestas múltiples y necesarias que actualmente cubren casi todos los espacios del conocimiento. Contar con recursos anexos ha permitido mejorar la infraestructura, contar con algún personal de apoyo, actualizar equipos, comprar materiales, colaborar con los gastos de investigación, dar un aporte a estudiantes para pasantías cortas, presentación de resultados en congresos o talleres... ${ }^{16}$

Esta preponderancia de lo docente sobre los otros ámbitos trata de equilibrarse mediante otras estrategias como la de régimen académico que privilegia las publicaciones -aunque toma en cuenta la obra didáctica y profesional-y los idiomas, sobre los otros rubros como serían la calidad docente y el trabajo en acción social.
El nuevo régimen de méritos, propuesto al Consejo Universitario y la categoría de "profesor postulante" busca una valoración más equilibrada de los académicos. Otro aspecto que parece relevante es el del prestigio. Los buenos docentes se quejan de que no se aprecia, ni reconoce su trabajo igual que el de los investigadores, tal vez por esta razón la Vicerrectoría de Docencia y la de Investigación proponen premios de reconocimiento que ya existen en el área administrativa.

Desde el nacimiento del SEP sus gestores tienen conciencia de la importancia de su relación con sus unidades base y de colaboración (Facultades y Escuelas) y de la necesidad de no "perjudicar" al grado quitándole los recursos más calificados, por eso y por la inconveniencia de gestar un grupo de elite dentro de la institución, plantean que el tiempo docente sea compartido entre cursos para estudiantes de grado y de posgrado.

En el ámbito de la investigación, por ejemplo, las comisiones de las escuelas continúan generando proyectos, programas y otras actividades que a menudo coordinan con la Vicerrectoría, pero es en los Centros, Institutos y Unidades Especiales de Investigación y SEP, donde se genera la mayor investigación y se establecen múltiples relaciones con el sector externo directamente a través de los investigadores o mediante la acción social ${ }^{17}$. Las Comisiones de Posgrado incluyen como miembro ex-oficio a directores y decanos y los directores de posgrado forman parte de los Consejos Asesores de las Escuelas y de los Consejos Científicos de los Centros e Institutos.

El SEP, como Sistema encabezado por un Consejo, la Decanatura y una oficina administrativo-académica coordinadora se rige, explícitamente, por los principios de calidad, pertinencia, ética, compromiso y colaboración interna y externa; la conciencia de estas premisas permite flexibilizar los aspectos académico administrativos (ciclos diferenciados o combinados, horarios, plazos, presentaciones fuera de la Universidad.... $)^{18}$ 
La polémica sobre el Sistema de Estudios de posgrado complejo y diverso, eficiente y generador de interrelaciones, resurge cada cierto tiempo como un juego de poder entre docencia e investigación (está adscrito como corresponde a esta última, pero en su Consejo participa también el Vicerrector de Docencia). Se discute la posibilidad de fragmentarlo insertando cada posgrado en una Escuela o Facultad ${ }^{19}$ ignorando, por una parte, los posgrados interdisciplinarios, regionales o interinstitucionales y por otra el cierre de posibilidades que abre el Sistema como red de vasos comunicantes con otras instancias nacionales e internacionales. La otra propuesta, polémica desde la creación, es la independencia total del posgrado en una Facultad de Graduados, lo que limitaría las interrelaciones y haría distinciones innecesarias entre académicos. El SISTEMA garantiza un trabajo de excelencia y procura constituirse en un espacio solidario que coordina y apoya.

\section{Atravesando disciplinas: aperturas, interrelaciones y resistencias}

La organización universitaria promueve, a nivel de Escuelas y Facultades el espacio disciplinario que, además, se apoya en "el principio de la departamentalización" ${ }^{20}$ que, a pesar de las tendencias actuales hacia lo multi, inter o transdisciplinario tiene muchos adeptos.

Desde hace varios años en diferentes foros de discusión y en documentos políticos y de políticas universitarias se habla de la necesidad de fomentar, promover y legitimar los trabajos en equipos, donde participen académicos de distintos ámbitos del conocimiento.

Esta propuesta discursiva encuentra los límites en reglamentos y normativas e incluso en nuestra idiosincrasia individualista y en la poca costumbre de compartir conocimientos con personas de otras áreas, aunque tenga los mismos intereses y objetivos. Es significativo el bajo reconocimiento en Régimen Académico a publicaciones compartidas y donde participan investigadores de diferentes disciplinas, por ejemplo.

Algunas de las políticas universitarias, sin embargo, trabajan en pro de esta propuesta que permite una visión más integral de los temas y problemas, al enfocarlos desde distintas aristas, con diversas teorías y métodos y diferentes perspectivas y experiencias, sobre todo, pero no exclusivamente en investigación, posgrado y acción social.

Los Centros de Investigación nacen como espacios inter o multidisciplinarios y es significativo que se multipliquen hasta 23, mientras los Institutos solo llegan a doce. Actualmente, sin embargo, la mayor parte de los Institutos cuentan con investigadores de varias disciplinas -más o menos afines- y sus proyectos revelan una vocación que trasciende lo disciplinario. El hecho de que, sin perder su base en las $\mathrm{Fa}$ cultades o Escuelas, queden adscritos a la Vicerrectoría de Investigación ${ }^{21}$, permite no solo que los problemas legalmente se diriman en su ámbito de pertinencia y pertenencia (no ya en docencia) sino que cuenten con más libertad para programar proyectos interdisciplinarios.

Varias de las revistas de la Universidad de Costa Rica responden a áreas del conocimiento, intereses temáticos o problemáticos, más que a disciplinas propiamente dichas y en ellas publican investigadores de diferentes especialidades o trabajos de equipo. Es el caso de la Revista Kañina, la de Biología Tropical, Ciencias Sociales, Gerontología, Educación o Intersedes, para solo nombrar algunas con diferentes características.

La organización de bases de datos con la información de los investigadores y las investigadoras, de los proyectos, programas, servicios y actividades de las unidades académicas de investigación por parte de la Vicerrectoría de Investigación (VI) y las bases de datos de los graduados con 
sus investigaciones o la de los reconocimientos, equiparaciones y convalidaciones de los títulos de posgrado provenientes del exterior del SEP permiten conocer el potencial académico con que se cuenta y partir de una investigación ya realizada por estudiantesprofesionales de distintos campos del saber.

La VI publica el Boletín Girasol (difusión electrónica y en papel) con actividades de investigación y el SEP el Boletín Notas (difusión electrónica); se publica la Guía informativa de Centros, Institutos y otras actividades de Investigación y se propone una imagen institucional (corporativa) de la investigación como una estrategia de comunicación, con logos específicos que identifican (unidad en la diversidad). Además, tanto la VI como el SEP cuentan con páginas electrónicas donde se puede consultar la información y establecer contactos. El SEP trabaja en este momento en un programa semanal para canal 15 (SEP-MILENIO) y en una propuesta de catálogo de los diferentes Posgrados ${ }^{22}$. Todo este esfuerzo comunicativo pretende forjar una imagen y un intercambio de actividades que estimulen la interrelación entre los investigadores y los posgrados entre sí y entre los posgrados y las investigaciones que se trabajan en Centros e Institutos.

Las Jornadas de Investigación institucionales, permiten conocer resultados de investigación y promueven la interrelación de investigadores de diferentes ámbitos del conocimiento que trabajan sobre temáticas o problemáticas comunes. Esto sin detrimento de las Jornadas que realizan las Escuelas, Centros e Institutos de manera particular.

En las Ferias o Exposiciones (EXPOUCR) se muestran los bienes y servicios con que la institución contribuye con la comunidad, muestra la relación con el sector externo y su incidencia en el mejoramiento de la calidad de vida.

En los Festivales de la Publicación, se hace entrega de las ediciones de la Editorial de la Universidad de Costa Rica (tanto libros, como compilaciones y revistas), como un estímulo a los académicos de los distintos campos del conocimiento que publican, individualmente o en equipo, los productos de sus investigaciones o de su creatividad. Este esfuerzo se complementa con la recopilación de otras publicaciones de todos los investigadores (que envían su información) realizadas externamente, tanto nacionales como internacionales.

En 1999, se inicia el trabajo de los Programas Institucionales de Investigación, promovidos por la Vicerrectoría, con el propósito de reunir a los investigadores interesados en un campo del conocimiento, desde su experiencia particular, con el propósito de dar respuesta a problemas de gran complejidad. Se cuenta con programas institucionales en "Ciencia e Ingeniería de Materiales", "Prevención y Mitigación de Desastres", "Gestión Municipal, participación ciudadana y desarrollo local”, "Drogas y Farmacodependencia". Los dos primeros grupos formulan, en este momento, programas de posgrado inter o transdisciplinarios; ya existe una Maestría académica interdisciplinaria en Farmacodependencia.

Desde 1957 la UCR opta por el humanismo con la concepción de un Sistema de Educación General ${ }^{23}$ a la base que comprende la Escuela de Estudios Generales con sus cursos de humanidades, repertorios, cultura y deporte y no responde a un criterio de "departamentalización", como tampoco lo hace el Sistema de Estudios de Posgrado (1975) que responde a una fuerte tendencia a lo interdisciplinario y contextual para apoyar la especialización.

En el Sistema de Estudios de Posgrado:

- $\quad$ existen varios programas que se clasifican en la modalidad de interdisciplinarios y que perfilan su plan curricular sobre el género, lo etario, una problemática o un ámbito del conocimiento de por sí multidisciplinario. 
Es el caso del Programa interinstitucional (UNA-UCR) en Estudios de la Mujer, los programas de posgrado en Gerontología, en Estudios Interdisciplinarios sobre Discapacidad, en Estudios Interdisciplinarios sobre Adolescencia y Juventud o en Ciencias Cognoscitivas.

- otros posgrados son interdisciplinarios por su naturaleza como Ciencias del Movimiento Humano, Ciencias Biomédicas, Diplomacia, Nutrición...

- $\quad$ los siete doctorados hasta ahora acreditados por CONARE responden a amplios espacios del conocimiento como Filosofía, Historia y Educación (pensamiento, proceso y formación); en Desarrollo Agrícola Tropical Sostenible, en Gobierno y Políticas Públicas, Sociedad y Cultura y Ciencias. Todos ellos reciben estudiantes de distintas disciplinas y cuentan con personal académico interdisciplinarios.

- $\quad$ con algunas dificultades se ha logrado compartir cursos, profesores extranjeros y proyectos de investigación entre varios campos. En principio hay planes de estudio que incluyen un porcentaje de créditos que pueden hacerse en otros posgrados y, se considera que las Maestrías son interdisciplinarias al menos por la procedencia de los estudiantes, mientras que las Especialidades tienden a ser para licenciados en el mismo campo.

\section{Nuevas tecnologías, educación permanente e impacto social}

La institución, en los últimos años, ha realizado un esfuerzo compartido con diferentes instancias para ampliar el campus y mejorar la infraestructura. La oficina a cargo de las construcciones (OEPI) ha logrado que los nuevos espacios que se construyen o remodelan sean funcionales y además estéticos. Basta con visitar los mini auditorios a través de todo el campus.

A este empuje se suma el mejoramiento de mobiliario y equipos de laboratorio y, sobre todo, la compra de equipos de cómputo y el cableado necesario para que prácticamente en cada rincón de la comunidad se pueda contar con acceso a internet. A la red internacional se suman las redes informáticas internas, las bases de datos y el mejoramiento del SIBDI y el DIEDIM integralmente ${ }^{24}$.

La Oficina Jurídica se preocupa por publicar los textos relevantes y se organiza de manera más adecuada para responder a las demandas continuas y las Oficinas de Registro y Administración Financiara están integradas en un proyecto piloto que busca agilizar los trámites garantizando la seguridad y la confiabilidad.

Desde la Rectoría se realiza un Diagnóstico Institucional (a cargo de la Magister Isabel González) que busca facilitar información para la toma de decisiones. En todo este proyecto de modernización de los recursos administrativos y actualización de los procesos interviene, desde luego, la capacidad y los programas informáticos. La Universidad se ha preocupado por tener la tecnología de punta necesaria para poder avanzar al ritmo de los tiempos y lo ha hecho de manera desconcentrada, dotando de equipo no solo a las oficinas administrativas sino promoviendo laboratorios en escuelas, facultades, bibliotecas para los usuarios estudiantes y académicos.

Este potencial académico y el mejoramiento de los equipos permiten el uso de las nuevas tecnologías de la información y la comunicación a nivel docente y de investigación. La apertura del Internet permite, por ejemplo, no solo la búsqueda de materiales actualizados sin el contacto rápido y directo con académicos y estudiantes de 
cualquier parte del mundo. Eso permite en el posgrado, contar con asesores de investigación externos, establecer contactos para intercambios académicos, para proyectos compartidos o para posibles. Permite un intercambio ágil, efectivo y abierto.

En los últimos años, diversas instituciones universitarias del exterior han querido venderle a la universidad posgrados semi presenciales que se darían parcialmente en sus instalaciones, posgrados a distancia con uno o dos encuentros y posgrados totalmente virtuales. Esta oferta también le ha llegado al SEP mediante la organización de posgrados virtuales en red, donde participan varias instituciones. Hasta el momento no se tienen políticas institucionales escritas, pero se trabaja con el criterio de que la UCR no es una universidad a distancia y que las propuestas compartidas se pueden aceptar si parten de criterios académicos de calidad y pertinencia y se negocia la participación y colaboración mutua.

En relación con el uso de las nuevas tecnologías en posgrado y los programas semi-presenciales la Universidad trabaja ya con un plan piloto en el Doctorado de Educación donde los estudiantes combinan seis semanas presenciales con diez a distancia. Siendo los doctorados eminentemente investigación, esta combinación de clases presenciales, con diálogo directo a través de internet ha dado excelentes resultados.

En relación con los cursos y actividades virtuales se asumen como complemento útil y necesario en los Posgrados que así lo consideren conveniente y se valoran como posibilidad viable y efectiva para los cursos especiales de posgrado y toda la gama de posibilidades que abre la educación para toda la vida o educación permanente. Coordinado por la Vicerrectoría de Acción Social (VAS) se plantea una propuesta para organizar, adecuadamente, el Sistema de Educación Continua. Existe actualmente un programa institucional entre la VI y la VAS que busca el mejoramiento de la vinculación con el sector externo.
En el caso del posgrado se han realizado enormes esfuerzos por aumentar el número, variedad y calidad de los cursos especiales de posgrado. La extensión y carácter de los cursos varía de acuerdo con intereses y necesidades y con ellos se pretende no solo profundizar en ciertos ámbitos del conocimiento, presentar novedades en investigación o actualizar teorías, métodos y técnicas sino también contribuir de manera ágil y eficiente con distintos procesos de transformación social. El SEP trata de garantizar el nivel académico y trabaja la parte de organización estrechamente relacionado con la VAS.

Un ejemplo de este esfuerzo lo constituyen los cursos de Gestión Local de la Salud o el de Epidemiología impartidos en distintas regiones del país por el posgrado en Salud Pública, en convenio con la Caja Costarricense de Seguro Social. El primero se imparte en 11 regiones del país con una duración de un año y para garantizar la calidad no solo se capacita a los instructores sino que se construyen 50 módulos escritos, algunos ya publicados en forma de libro. Actualmente se cuenta con más de 1000 graduados provenientes de diferentes disciplinas.

Varios de estos cursos especiales de posgrado son impartidos por académicos extranjeros de alto nivel y, actualmente, se empiezan a planear como educación a distancia. Estos cursos y otras actividades están siendo reconocidos para el curriculum profesional y para procesos de acreditación y recertificación. Tal es el caso del Colegio de Médicos y Cirujanos.

La investigación que se realiza en las Especialidades y en las Maestrías profesionales, al ser práctico aplicada, busca resolver problemas más concretos y permite un impacto social a corto o mediano plazo. En algunos de estos espacios se busca presentar los trabajos a los grupos directamente involucrados o que pueden aprovechar los resultados, lo mismo que divulgarlos por medios más accesibles que las revistas especializadas. 


\section{Por el camino del estímulo y la calidad}

Como no es posible profundizar en los cambios cualitativos que se han dado en este último quinquenio en la UCR, nos limitaremos, como apertura para nuevos diálogos, a sintetizar algunas decisiones que garantizan un mejor funcionamiento, mayor calidad e impacto en investigación y posgrado.

- La política de descongelamiento de plazas para apoyar estudiantes que se encuentran en el exterior o de docentes que hacen sus estudios de posgrado en el Sistema de la UCR.

- La reorganización interna de la VI y del SEP para lograr un trabajo más eficiente y menos centralizado y el fortalecimiento de mecanismos de autoevaluación y evaluación permanente, con propósitos de actualización y mejoramiento académico.

- La VI forma varias comisiones de académicos con excelentes resultados: Comité ético científico y Comisión de Evaluación (1998), Comisión de Comunicación (1999), Comisión de Biodiversidad (1999), Comisión de Biotecnología.

- $\quad$ La readecuación de la UTT (Unidad de Transferencia Tecnológica), por ejemplo, se efectúa para que colabore con los investigadores en el espacio de la propiedad intelectual, las patentes y las relaciones con el sector productivo. Se organiza la Red de Gestores de Vinculación a partir de proyectos interdisciplinarios y la Red de Gestores de Cooperación nacional e internacional (2000), con representantes de cada Centro e Instituto.

- Fortalecimiento de redes con universidades extranjeras (redes Alfa) e inserción y fortalecimiento con la participación en organismos internacionales de educación superior (OUI, AUIP, CILPIC, ANUIES-CSUCA, CSUCA...)

- $\quad$ Creación de Programas Institucionales de Investigación que fortalecen los posgrados y otras actividades interdisciplinarias.

- $\quad$ El ordenamiento de la información en bases de datos accesibles que permitan más interrelaciones entre los espacios y una mejor toma de decisiones (en los últimos cinco años en el SEP se pasa de un 10 a casi un $80 \%$ de datos incluidos en bases de datos en la red informática y se mejoran notablemente los equipos y sistemas de información).

- $\quad$ El resurgimiento, mejoramiento y/o fortalecimiento de programas institucionales de la envergadura de las Jornadas de Investigación (octubre 1994, agosto 1997 y agosto 1999), las EXPO-UCR (noviembre 1998 y agosto 2001), los Festivales de la Publicación (anual), la Feria Vocacional (el SEP participa en los últimos cuatro años). En el año 2000 se realiza por primera vez el Congreso de Investigación (CONINVES) entre las universidades públicas.

- Se amplían y mejoran los medios de comunicación que informan sobre la investigación y el posgrado. Como un ejemplo relevante están el Boletín Girasol, los suplementos de graduación de posgrado en La Nación o el programa SEP-MILENIO (Canal 15).

- Una fuerte participación en las distintas instancias de CONARE, del CONICIT y de otras instancias nacionales de fortalecimiento a la educación. 
- Los concursos de antecedentes para profesores en propiedad se analizan con criterios que buscan evitar abusos y fundamentar las elecciones de las Asambleas; además se aprueba la categoría de Profesor Postulante que busca dar seguimiento a los interinos y garantizar una escogencia de académicos con proyección a futuro.

- $\quad$ Se plantea una reforma en Régimen Académico y se propone un régimen de méritos para los académicos aún jóvenes y se propone una escala que favorezca con salarios más competitivos, a los nuevos profesionales que ingresan a la institución.

- A partir del 2001, se exige el título de posgrado para concursar por un puesto en propiedad y para optar por la dirección de un Centro, Instituto, Unidad Especial o dirigir una Revista Académica. Estas medidas obligan a los académicos universitarios a mejorar su competencia y actualizarse y condiciona a quienes quieren ingresar a formar parte de los universitarios.

- Se abre la categoría de Catedrático a cinco y ahora siete pasos académicos (nueve puntos cada uno), favoreciendo la productividad y se toman iniciativas en dos sentidos: por una parte se propone la figura de un $\mathrm{Ca}$ tedrático de la Educación Superior Costarricense, que pueda trabajar como tal en todas las instituciones públicas (CONARE) y, por otra, la de un Catedrático Extraordinario que por méritos de producción académica, pueda gozar de un año sabático completo, el cargo de profesor emérito cuando se jubile y un aumento salarial.

- La Oficina de Asuntos Internacionales y Cooperación Externa fortalece y diversifica el apoyo a los becarios al exterior, pasantías cortas para docentes, académicos extranjeros, redes y consorcios y se manejan con más pertinencia y seguimiento los Convenios Internacionales. Estas funciones y otras más son apoyadas desde la VI y desde el SEP que buscan favorecer el espacio de la investigación.

- Como una forma de apoyar la Educación permanente o "para toda la vida" el Consejo del SEP toma dos acuerdos relevantes. Por una parte, establece la apertura para que todo graduado del Sistema pueda continuar empadronándose y llevando cursos en diferentes Posgrados y, por otra, establece la posibilidad de que las Comisiones reconozcan cursos de un programa conducente a título para completar otro posgrado.

Políticas transparentes de trabajo académico, rendición social de cuentas, respuestas reales y efectivas a las demandas y necesidades sociales, generación de conocimientos y una búsqueda constante de calidad que permita incidir en el mejoramiento de la calidad de vida de la comunidad nacional y regional colocan a la Universidad de Costa Rica como una institución de educación superior pionera, transformadora y moderna que conserva su vocación de instancia pública, de servicio y basa sus estrategias en la excelencia académica, el acceso y la participación, el humanismo y la ética.

\section{Notas}

1. Decana del Sistema de Estudios de Posgrado, Universidad de Costa Rica.

2. Hotel Legend, Nicaragua (19 al 21 de noviembre, 2001). Realizado bajo el auspicio del Programa ANUIES-CSUCA y las universidades públicas nicaragüenses (UNAN-Managua, 
UNAN-LEON, UNI y UNA). La coordinación estuvo a cargo del Director de posgrado de la UNAN-MGA, Dr. Julio Traña.

3. La Red Helsinki-España y el Proyecto Pacífico realizan en la Universidad de Costa Rica (junio 2002) un taller sobre la sociedad del conocimiento y el impacto de las nuevas tecnologías donde, al menos en teoría, pone énfasis en el poder del conocimiento como el eje de nuestro siglo.

4. En el VI Congreso Universitario, cuya primera fase se desarrolla entre el 13 y el 17 de mayo y se continúa entre el 4 y el 6 de junio, se plantean 48 ponencias la mayor parte de las cuales corresponden a estructura y gobierno. Aquellas que presentan cambios radicales en relación con las Vicerrectorías, el Consejo Universitario y el Sistema de Estudios de Posgrado son votadas en contra y no serán objeto de discusión en la segunda etapa, a la que asistirán los que estuvieron presentes en un $60 \%$ de las sesiones.

5. En el VI Congreso Universitario (junio y agosto 2002) algunas de las ponencias tratan de favorecer la inclusión de grupos con menos oportunidades.

6. Los doctorantes deben realizar una pasantía de un mínimo de un ciclo lectivo fuera del país.

7. Es claro que de acuerdo con el título y la especificidad del área de conocimiento, la investigación es diferente. Teórica, práctico aplicada, creativa, epidemiológica, estudios de caso...

8. La UCR tiene acreditados como regionales centroamericanos los Programas de Administración Pública, Estudios Interdisciplinarios sobre Discapacidad, Microbiología, Química, Biología, Sociología, Ciencias Políticas, Suelos, Doctorado en Desarrollo Agrícola Tropical Sostenible, Doctorado en Educación, Posgrado en Geología, en Historia, en Geografía, Ciencias Biomédicas y se encuentran en proceso de acreditación Artes, Ciencia de Alimentos, Administración Universitaria, Comunicación, Filosofía y Gerontología.

9. La Vicerrectoría de Vida Estudiantil, bajo la dirección de la Dra. Ligia Bolaños Varela, realiza en 1999, un concienzudo diagnóstico y presenta una propuesta de rediseño que valora al estudiante integralmente y propone una estructura administrativa más coherente con una Universidad de futuro, la Asamblea Colegiada Representativa lo rechaza, después de una dura campaña de desprestigio.

10. Artes y Letras, Ciencias Básicas, Ciencias Sociales, Ciencias de la Salud e Ingenierías y Agronomía. Esta última se divide en dos cuando la Asamblea Colegiada Representativa, en setiembre del 2001, aprueba el Área Agroalimentaria.

11. Existen Facultades integradas como Derecho, Odontología, Farmacia o Microbiología y otras divididas en Escuelas como Ciencias Sociales, Educación o Ciencias Económicas, por ejemplo.

12. Tres Vicerrectorías académicas: Investigación, Docencia y Acción Social; una Vicerrectoría de Vida Estudiantil y una de Administración.

13. Actualmente compuesto por el Rector, representantes de las áreas académicas, un representante de colegios profesionales, dos estudiantes y el Ministro de Educación.

14. En el presupuesto del 2002 se consolida un apoyo extraordinario, para los Doctorados Académicos y se trabaja con la Rectoría y la OAFCI una propuesta que mejore las becas y las posibilidades de pasantías al exterior.

15. El Fondo Solidario 170 ha establecido como prioridad, en estos primeros años, el mejoramiento en infraestructura y equipos y el apoyo a la investigación de estudiantes y docentes del exterior.

16. El Consejo Universitario aprueba los lineamientos para el trabajo en 1993 y en el 2001 los actualiza por dos años, solicitando una serie de estudios y regulaciones.

17. Un análisis comparativo entre la investigación de la UCR antes y después de esta organización evidencia, claramente, su éxito.

18. Nuestro lema es que en la oficina coordinadora estamos para resolver problemas y no para transferirlos. Creemos en la agilidad, la eficiencia y en el trato amable y personalizado.

19. En el VI Congreso Universitario varias ponencias plantean esta posibilidad. Todas ellas fueron rechazadas por la Comisión plenaria y no pasan a la segunda etapa. 
20. El principio de departamentalización no existe para posgrado y, en principio, las maestrías son interdisciplinarias.

21. Reforma al Estatuto Orgánico, 2001.

22. Se trabaja en una imagen para la documentación de cada Programa de Posgrado que, con unidad en la diversidad, se proyecta a largo plazo. Con ella se harán los desplegables, afiches, página weeb...

23. En "Políticas prioritarias para la formulación y ejecución del plan presupuesto de la UCR para el año 2001", mayo 2001, se demanda "Lograr la continuidad en la formación humanística universitaria mediante el intercambio articulado entre el Sistema de Estudios de Posgrado y el Sistema de Educación General, en las diversas actividades académicas que ambos lleven a cabo". Art. 4.8, p. 14 .

24. Desaparece la Oficina de Publicaciones, se invierte en la Editorial, en la Librería Universitaria, en la compra de material bibliográfico actualizado y en el mejoramiento de las condiciones de espacio físico para las colecciones universitarias. Los libros de texto Serie: Hacia el siglo XXI, elaborados por más de una cincuentena de académicos, para escuelas y colegios, han resultado muy exitosos.

\section{Referencias bibliográficas}

Arrien, Juan Bautista y otros. La educación y la reforma de la educación en cinco países centroamericanos. Nicaragua: Editorial UCA, 1999.

De Castilla Urbina, Miguel y otros. Palabra de Maestro. Nicaragua: IDEUCA, 2001.

Delors, Jacques y otros. La educación encierra un tesoro. Madrid: Edic. UNESCO, 1996.

Didriksoon, Axel. La Universidad de la Innovación. Una estrategia de transformación para la construcción de universidades del futuro. Venezuela: IESALC-UNESCO, 2000.

González García, Yamileth y Pérez Yglesias, María. "Hacia una Universidad Cualitativa: Los posgrados en Ciencias Sociales". En: Revista de CIENCIAS SOCIALES, Universidad de Costa Rica, No. 40, 1994.

Morin, Edgard. Los siete saberes necesarios a la educación del futuro. Venezuela: IESALC-UNESCO, 2000.

Ruiz, Ángel. El siglo XX y el papel de la Universidad; El destino de Costa Rica y la Educación Superior y La Educación Superior en Costa Rica. San José: Editorial UCR, 2002.

Tünnermann, Carlos. La Educación Superior frente al cambio. Costa Rica: EDUCA/CSUCA, 1997.

. La Educación en el horizonte del siglo XXI. Venezuela: IESALCUNESCO, 2000.

. Universidad y Sociedad. Nicaragua: HISPAMER, 2001.

Yarzábal, Luis. Consenso para el cambio de la educación superior. Venezuela: IESALC- UNESCO, 1999.

\section{Informes, ponencias y documentos}

VI Congreso Universitario. "Ponencias y otros documentos complementarios". Auditorio Abelardo Brenes Córdoba, Universidad de Costa Rica. Mayo, junio y agosto 2002.

Consejo Universitario. Políticas prioritarias para la formulación y ejecución del plan-presupuesto de la Universidad de Costa Rica para los años 1999 al 2002. Editorial UCR. 
Documentos de Comisiones provisionales. Proyectos de Programas de Posgrado, presentados para su aprobación en el SEP y en CONARE.

Documentos I Seminario del Sistema de Estudios de Posgrado, Universidad de Costa Rica. Colegio de Ingenieros y Arquitectos, 1984. (Decano Dr. Oscar Fernández).

Documentos II Seminario del Sistema de Estudios de Posgrado, Universidad de Costa Rica. Colegio de Ingenieros y Arquitectos, 1993. (Decano Dr. Luis Camacho).

Documentos Jornadas de Autoevaluación del Sistema de Estudios de Posgrado, UCR, Estación Experimental Alfredo Volio Mata, 1995 (Decana Yamileth González G.).

Documentos Jornadas de Reflexión del Sistema de Estudios de Posgrado, UCR, Estación Experimental Alfredo Volio Mata, 2000 al 2002 (Decana Dra. María Pérez Y).

Documentos varios direcciones de Maestrías (Reglamentos Internos, Informes, Listas de tesis...) y Oficina Coordinadora del Sistema de Estudios de Posgrado.

. Documentos de los Archivos del Consejo Universitario referentes al Sistema de Estudios de Postrado.
Documentos varios de las Vicerrectorías de Investigación, Docencia y Acción Social.

"Información necesaria para el estudio de una carrera de posgrado". CONARE, 1976.

"Normas para la apertura de nuevos programas de posgrado". Consejo del SEP, 1986.

\section{OPES}

"Informe Crítico sobre los Programas de Posgrado en las Instituciones de Educación Superior Universitaria”, Opes, 1985.

"Desarrollo de los programas de posgrado en las instituciones de educación superior costarricenses", 1993.

PNUD Informe Estado de la Región en Desarrollo Humano Sostenible. Costa Rica: PNUD, 1999.

Reglamento Sistema de Estudios de Posgrado. Universidad de Costa Rica, 1975.

Propuesta actual en trámite, en el Consejo Universitario, 1998, UCR.

Red Helsinski-España. La sociedad del conocimiento y los derechos humanos. Encuentro Internacional Universitario, Universidad de Costa Rica. 21 al 28 de junio 2002.

María Pérez Yglesias Decana del Sistema de Estudios de Posgrado (SEP) Universidad de Costa Rica 